\title{
Introduction: special issue of selected papers of ACML 2013
}

\author{
Cheng Soon Ong ${ }^{1}$. Wray Buntine ${ }^{2}$. Tu-Bao $\mathrm{Ho}^{3}$. \\ Masashi Sugiyama ${ }^{4}$ - Geoffrey I. Webb ${ }^{2}$
}

Published online: 27 March 2015

(C) The Author(s) 2015

The 5th Asian Conference on Machine Learning (ACML 2013) was held on 13-15 November 2013, at the Australian National University, Canberra, Australia. ACML aims at providing a leading international forum for researchers in machine learning and related fields to share their new ideas and achievements. The conference called for research papers reporting original investigation results, and received 103 paper submissions from 22 countries. We eventually accepted 32 papers with 13 long presentations and 19 short presentations. Every paper was double-blind reviewed by at least 3 expert reviewers, and these were then used to form the opinion of 2 senior program committee members (Ong and Ho 2013).

Authors of the top papers were invited to submit a significantly extended version to this special issue. The selection was made by the team of guest editors consisting of Program Chairs, General Chairs and Steering Committee Chairs, on the basis of the scientific quality and potential impact of these papers, as indicated by the conference reviewers and quality of the presentations and posters. These extended papers have been reviewed again for two or three rounds of peer-review process according to the journal criteria and for each paper some

$\triangle$ Cheng Soon Ong

Cheng-Soon.Ong@nicta.com.au

Wray Buntine

wray.buntine@monash.edu

Tu-Bao Ho

bao@jaist.ac.jp

Masashi Sugiyama

sugi@cs.titech.ac.jp

Geoffrey I. Webb

geoff.webb@monash.edu

1 Machine Learning Research Group, NICTA, Canberra, ACT 2601, Australia

2 Faculty of Information Technology, Monash University, Clayton, VIC 3800, Australia

3 School of Knowledge Science, Japan Advanced Institute of Science and Technology, Ishikawa, Japan

4 Graduate School of Frontier Sciences, The University of Tokyo, Tokyo 113-0033, Japan 
additional reviewers have been involved to enhance the journal review process. Finally, we present the following six papers in this special issue.

The paper "Generalized Aitchison Embeddings for Histograms" by Tam Le and Marco Cuturi propose a method for computing distances between histograms on a probability simplex. They suggest algorithms that build on metric learning methods to estimate parameters of a generalized class of Aitchison Embeddings. The resulting non-convex optimization problem is solved using alternating optimization approaches and accelerated gradient descent approches.

The paper "Information Retrieval Approach to Meta-visualization" by Jaakko Peltonen and Ziyuan Lin propose a meta-visualization method to analyse multiple visualizations corresponding to various aspects of a data analysis task. They extend the recently proposed formalization of visualization as an information retrieval task which allow the performance of visualization methods to be quantified. Based on this, they proposed a nonlinear embedding of multiple plots on a display such that similar visualizations are close together.

The paper "Multilabel Classification through Random Graph Ensembles" by Hongyu $\mathrm{Su}$ and Juho Rousu, propose a structured output prediction based method for multilabel classification. Using an ensemble of classifiers, each constructed by considering a random graph to represent the structure, they investigate the effect of diversity and coherence of the base models. The proposed method is shown to compare favourably on a set of heterogenous benchmark problems against multilabel AdaBoost, convex multitask feature learning, and single target learning approaches.

The paper "Random Projections as Regularizers: Learning a Linear Discriminant from Fewer Observations than Dimensions" by Robert John Durrant and Ata Kaban show theoretical guarantees for an averaging-ensemble of randomly projected Fisher Linear Discriminant classifiers. Focusing on the high dimension low sample size regime, they derive the exact form of the generalization error of the ensemble, giving results linking the performance of the ensemble to that of the corresponding linear discriminant learned in the full data space. A preliminary version of this work received the best paper award at the 5th Asian Conference on Machine Learning.

The paper "Linearized Alternating Direction Method with Parallel Splitting and Adaptive Penalty for Separable Convex Programs in Machine Learning” by Zhouchen Lin, Risheng Liu and Huan Li proposes a multi block extension to Linearized Alternating Direction Method (LADM) for separable convex programs. Many machine learning problems can be expressed as separable convex programs, and the proposed method is particularly useful for sparse representation and low-rank recovery problems because its subproblems have closed form solutions and the sparsity and low-rankness of the iterates can be preserved during the iteration. Furthermore, the proposed method is well suited to modern distributed computing architectures due to its parallel nature.

The paper "Unconfused Ultraconservative Multiclass Algorithms" by Ugo Louche and Liva Ralaivola proposes a multiclass generalisation of learning linear classifiers from noisy datasets. Motivated by a weighted average of points from the noisy training set, they consider the multiclass setting. They directly generalise classification noise by a confusion matrix, and propose a theoretically well founded Unconfused Multiclass Additive Algorithm.

This special issue would not have been possible without the contributions of many people. We wish to thank all the authors for submitting their papers to this special issue. We also with to express our gratitude to all the referees for the time and effort in ensuring the quality of the submissions for this issue. We also wish to thank Melissa Fearon, Melissa Warren, and Sudha Subramaniam from the Springer team for their assistance throughout the organisation and production of this special issue. 


\section{References}

Ong, C. S., \& Ho, T. -B. (Eds.). (2013). JMLR Workshop and Conference Proceedings, vol. 29. Asian Conference on Machine Learning. http://jmlr.csail.mit.edu/proceedings/papers/v29/ 